\title{
Minimum Mean Squared Error Interference Alignment
}

\author{
David A. Schmidt*, Changxin Shi ${ }^{\dagger}$, Randall A. Berry ${ }^{\dagger}$, Michael L. Honig ${ }^{\dagger}$ and Wolfgang Utschick* \\ *Associate Institute for Signal Processing \\ Technische Universität München, 80290 Munich, Germany \\ Email: \{dschmidt,utschick\}@tum.de \\ ${ }^{\dagger}$ Department of Electrical Engineering and Computer Science \\ Northwestern University, Evanston, Illinois 60208, USA \\ Email: cshi@u.northwestern.edu, \{rberry,mh\}@eecs.northwestern.edu
}

\begin{abstract}
To achieve the full multiplexing gain of MIMO interference networks at high SNRs, the interference from different transmitters must be aligned in lower-dimensional subspaces at the receivers. Recently a distributed "max-SINR" algorithm for precoder optimization has been proposed that achieves interference alignment for sufficiently high SNRs. We show that this algorithm can be interpreted as a variation of an algorithm that minimizes the sum Mean Squared Error (MSE). To maximize sum utility, where the utility depends on rate or SINR, a weighted sum MSE objective is used to compute the beams, where the weights are updated according to the sum utility objective. We specify a class of utility functions for which convergence of the sum utility to a local optimum is guaranteed with asynchronous updates of beams, receiver filters, and utility weights. Numerical results are presented, which show that this method achieves interference alignment at high SNRs, and can achieve different points on the boundary of the achievable rate region by adjusting the MSE weights.
\end{abstract}

\section{INTRODUCTION}

The number of non-interfering data streams in a network with interfering nodes depends on the extent to which the interference can be aligned at each receiver. For example, with $N \times N$ multi-input multi-output (MIMO) channels, it has been shown that the number of non-interfering data streams can be as many as $2 N-1$ [1].

We consider a peer-to-peer network with $K$ interfering MIMO links, where the performance of each transmitterreceiver pair is measured by a utility function, which depends on the achievable rate. Our problem is to determine the precoders that maximize the sum utility over all users. For this purpose we seek a distributed algorithm with limited information exchange among the nodes. To simplify the problem we will assume that each precoding matrix has rank one, corresponding to beamforming, the receivers are linear, and that the interference is treated as noise, so that the rate is determined by the received Signal-to-Interference Plus Noise Ratio (SINR).

At high SNRs the optimal set of transmitted beams give received beams that are aligned at the receivers. Although

This work was supported by ARO under grant W911NF-06-1-0339 and DARPA under grant W911NF-07-1-0028. general conditions for alignment are relatively easy to specify, given complete channel knowledge for the entire network, computing the optimal aligned solution can still be challenging when $K$ is large. This is because the number of aligned solutions grows rapidly with the network size, making it difficult to choose the best solution. Furthermore, for low to moderate SNRs alignment does not generally maximize sum utility, due to the associated loss in degrees of freedom.

One approach to distributed resource allocation in a peerto-peer network has been proposed in [2], [3], and is based on the exchange of interference prices. Given a set of announced prices, a transmitter selects a resource (e.g., power or beam) to maximize its utility minus interference cost to other users. This approach is used in [4] to optimize precoding matrices for two interfering users, and is observed to achieve near-optimal performance. However, we present an example with three users, which indicates that this approach typically does not achieve alignment at high SNRs. This is due to the behavior of the sum rate objective, which exhibits a very steep maximum at the optimal (aligned) solution.

We consider an alternative approach to sum utility maximization based on weighted Minimum Mean Squared Error (MMSE) beamforming. The MMSE criterion is observed to transform the rate objective into a "better-behaved" objective with a broader minimum. MMSE beam updates can be iterated with receiver updates asynchronously, and numerical results are presented, which indicate that this algorithm finds aligned solutions at high SNRs, and matches the performance of interference pricing at lower SNRs. This algorithm is distributed in the sense that each transmitter must know only its direct channel matrix and combined cross-channel-receiver gains to neighboring receivers (same as for the pricing algorithm).

The sum MMSE approach to beamforming is compared with the "Max-SINR" algorithm for precoder optimization recently proposed in [5]. We observe that the Max-SINR updates are closely related to the sum MMSE beam updates, the difference being in how the beams are scaled to satisfy a power constraint. The Max-SINR and sum MMSE algorithms are observed to achieve essentially the same performance for the examples considered. Attractive properties of the sum 
MMSE algorithm are that it has provable convergence, and it can be easily modified to account for different user priorities, or utilities, by introducing MSE weights. ${ }^{1}$

To maximize a sum utility objective, the MSE weights can be adapted according to the user utilites. This leads to a twostage algorithm in which the beams are adapted in an inner loop with fixed weights, followed by weight updates to match the current operating point. Conditions on the utility functions are given, which guarantee that the sum utility converges. Numerical results are presented for three users, which show different points in the rate region corresponding to different MSE weights.

\section{SySTEM MOdEL}

We consider an interference network consisting of $K$ transmitter-receiver pairs; we refer to each pair as a user. The receiver for user $k$ only decodes the signal from transmitter $k$ and treats interference from all other transmitters as noise. Each transmitter and receiver has $N$ antennas. The channel matrix $\boldsymbol{H}_{j k} \in \mathbb{C}^{N \times N}$ contains the complex channel gains between the $N$ antennas of transmitter $k$ and the $N$ antennas of receiver $j$.

Even though each transmitter-receiver pair is a MIMO link, we do not consider multiplexing, i.e., each user transmits only one beam. The beamforming vector used by transmitter $k$ is denoted as $\boldsymbol{v}_{k} \in \mathbb{C}^{N}$ and is subject to the transmit power constraint $\left\|\boldsymbol{v}_{k}\right\|_{2}^{2} \leq 1$. Furthermore, we assume linear receivers, i.e. the receiver of user $k$ forms the symbol estimates

$$
\hat{s}_{k}=\boldsymbol{g}_{k}^{\mathrm{H}} \boldsymbol{H}_{k k} \boldsymbol{v}_{k} s_{k}+\sum_{\substack{j=1 \\ j \neq k}}^{K} \boldsymbol{g}_{k}^{\mathrm{H}} \boldsymbol{H}_{k j} \boldsymbol{v}_{j} s_{j}+\boldsymbol{g}_{k}^{\mathrm{H}} \boldsymbol{n}_{k}
$$

where $s_{j}$ is the unit variance data symbol of transmitter $j$ (intended for receiver $j$ ) and $\boldsymbol{g}_{k}$ is the linear receive filter of user $k$. The additive noise $\boldsymbol{n}_{k}$ is assumed to be white with covariance matrix $\mathrm{E}\left[\boldsymbol{n}_{k} \boldsymbol{n}_{k}^{\mathrm{H}}\right]=\sigma^{2} \mathbf{I}$.

Each user's overall performance is given by a utility $u_{k}\left(\gamma_{k}\right)$ that is an increasing function of the SINR

$$
\gamma_{k}=\frac{\left|\boldsymbol{g}_{k}^{\mathrm{H}} \boldsymbol{H}_{k k} \boldsymbol{v}_{k}\right|^{2}}{\sum_{j \neq k}\left|\boldsymbol{g}_{k}^{\mathrm{H}} \boldsymbol{H}_{k j} \boldsymbol{v}_{j}\right|^{2}+\left\|\boldsymbol{g}_{k}\right\|_{2}^{2} \sigma^{2}}=\frac{S_{k}}{I_{k}+N_{k}} .
$$

The problem is to maximize the sum utility over the beamforming vectors $\boldsymbol{v}_{k}$ 's and receive filters $\boldsymbol{g}_{k}$ 's:

$$
\max _{\substack{\boldsymbol{v}_{1}, \ldots, \boldsymbol{v}_{K} \\ \boldsymbol{g}_{1}, \ldots, \boldsymbol{g}_{K}}} \sum_{k=1}^{K} u_{k}\left(\gamma_{k}\right) \quad \text { s.t.: } \quad\left\|\boldsymbol{v}_{k}\right\|_{2}^{2} \leq 1 \quad \forall k \in\{1, \ldots, K\} .
$$

Example utility functions include the achievable data rate $u_{k}\left(\gamma_{k}\right)=\log \left(1+\gamma_{k}\right)$ and the " $\alpha$-fair" utility $\gamma_{k}^{\alpha}$.

\footnotetext{
${ }^{1}$ Proving convergence of the Max-SINR algorithm appears to be more difficult due to the lack of a specified global objective.
}

\section{A. Interference Alignment}

In the regime of asymptotically high SNR, i.e., as $\sigma^{2} \rightarrow 0$, it is generally optimal to avoid interference completely so that

$$
\boldsymbol{g}_{k}^{\mathrm{H}} \boldsymbol{H}_{k j} \boldsymbol{v}_{j}=0 \quad \forall(k, j) \quad k \neq j .
$$

For random i.i.d. channels, this is conjectured to be possible without shutting off users (i.e., $\boldsymbol{v}_{k} \neq \mathbf{0}$ and $\boldsymbol{g}_{k} \neq \mathbf{0} \forall k \in$ $\{1, \ldots, K\})$ with high probability as long as $K \leq 2 N-1$ [6].

Let us examine the "fully loaded" case $K=2 N-1$. According to (4), all interference coming from the $K-1$ unintended transmitters must be orthogonal to the $N$-dimensional receive filter vector. Consequently, the $K-1=2 N-2$ interference components must be aligned in an $(N-1)$ dimensional subspace at each receiver, leaving one dimension free for identifying the intended signal [1].

While for two antennas $(N=2)$ it is possible to compute the solutions to (4) explicitly, finding aligned solutions by solving (4) directly appears to be intractable for $N>2$. A natural approach to finding such a solution would be to apply a gradient algorithm to (3) in the high SNR regime. This turns out to be problematic, since for the sum rate utility, the "good" local optima corresponding to aligned solutions have very narrow regions of attraction, and are not easily found by following the direction of the steepest ascent from arbitrary initializations. Instead, a gradient algorithm typically converges to another local optimum with either substantial interference, or with a fewer number of active users (below $2 N-1)$.

\section{B. Distributed Algorithms}

In [5], an iterative Min Leakage algorithm is proposed that converges toward an aligned solution from arbitrary initializations, if alignment is possible. This can be used to solve (3) for high SNRs when $K \leq 2 N-1$. Furthermore, this algorithm is distributed in that it requires the nodes to exchange limited information. Our focus is on distributed algorithms for solving (3) across a range of SNRs. In particular, for finite SNR, the conditions in (4) may not be optimal, i.e., tolerating some interference may be advantageous.

A distributed algorithm for beam allocation, based on the exchange of interference prices, has been proposed in [3], [4]. This has been shown to perform well for MultipleInput/Single-Output (MISO) interference networks as well as for two-user MIMO interference channels [4]. For MIMO interference networks, it operates as follows: in each iteration, receiver $k$ announces an interference price

$$
\pi_{k}=-\frac{\partial u_{k}\left(\gamma_{k}\right)}{\partial I_{k}}
$$

to all transmitters, which is the marginal decrease in utility for an increase in received interference. The transmitters asynchronously update their beams to maximize their own utility minus the "cost" of the interference they produce, i.e., they solve

$$
\max _{\boldsymbol{v}_{k}} u_{k}\left(\gamma_{k}\right)-\sum_{j \neq k} \pi_{j}\left|\boldsymbol{g}_{j}^{\mathrm{H}} \boldsymbol{H}_{j k} \boldsymbol{v}_{k}\right|_{2}^{2} \quad \text { s.t: } \quad\left\|\boldsymbol{v}_{k}\right\|_{2}^{2} \leq 1 .
$$




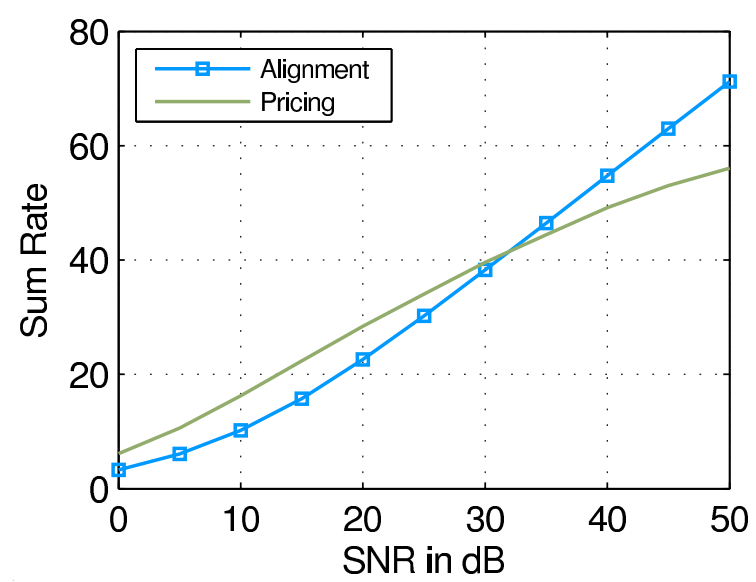

Fig. 1. Sum rate versus SNR for interference pricing and alignment.

A stationary point of this algorithm can be shown to fulfill the Karush-Kuhn-Tucker (KKT) conditions of the optimization in (3).

Figure 1 shows an example of the performance of this algorithm in a MIMO network with $K=3$ users and $M=2$ antennas at each transmitter and receiver. Each user $k$ has a rate utility function $u_{k}\left(\gamma_{k}\right)=\log _{2}\left(1+\gamma_{k}\right)$. The plot shows the average sum rate in bits per channel use versus SNR, defined as $1 / \sigma^{2}$. The performance is averaged over 100 channel realizations where the channel coefficients are i.i.d. complex Gaussian with unit variance. Also, shown is the sum rate achieved by an aligned solution obtained from the Min Leakage algorithm. It can be seen that for low SNRs pricing performs better, while for higher SNRs pricing does not achieve the optimal slope and performs worse than the aligned solution. As with a (centralized) gradient algorithm, it appears that at high SNRs, the pricing algorithm is attracted to non-aligned local optima. ${ }^{2}$

In [5], a second distributed algorithm called the MaxSINR algorithm was also proposed. This is a variation of the Min Leakage algorithm, modified with the aim of finding a "good" set of beams for finite SNR. The Max-SINR algorithm iteratively exchanges the role of transmitters and receivers in the network; in the reverse direction, the beamformers act as receive filters and the receive filters are used as beamformers. The update procedure consists of computing the receive filters that maximize the received SINR. In the original direction of communication, the receive filter update for user $k$ is

$$
\boldsymbol{g}_{k}=\theta_{k}\left(\sum_{j} \boldsymbol{H}_{k j} \boldsymbol{v}_{j} \boldsymbol{v}_{j}^{\mathrm{H}} \boldsymbol{H}_{k j}^{\mathrm{H}}+\sigma^{2} \mathbf{I}\right)^{-1} \boldsymbol{H}_{k k} \boldsymbol{v}_{k}
$$

with $\theta_{k}$ such that $\left\|\boldsymbol{g}_{k}\right\|_{2}^{2}=1$, and the beamformer update is

$$
\boldsymbol{v}_{k}=\beta_{k}\left(\sum_{j} \boldsymbol{H}_{j k}^{\mathrm{H}} \boldsymbol{g}_{j} \boldsymbol{g}_{j}^{\mathrm{H}} \boldsymbol{H}_{j k}+\sigma^{2} \mathbf{I}\right)^{-1} \boldsymbol{H}_{k k}^{\mathrm{H}} \boldsymbol{g}_{k}
$$

\footnotetext{
${ }^{2}$ Indeed, the interference prices essentially convey gradient information to each user.
}

where $\beta_{k}$ is chosen to fulfill $\left\|\boldsymbol{v}_{k}\right\|_{2}^{2}=1$.

Numerical results in [5] show that this algorithm has good performance in terms of sum-rate over a range of SNR values. However, no convergence proof is given and it is not clear how to modify it to account for other utility functions. Next, we introduce a closely-related distributed algorithm based on minimization of the mean squared error (MSE) to addresses these difficulties. In particular, it is straightforward to show that this algorithm converges. Moreover, we extend the algorithm to accommodate different utility functions.

\section{MSE MiNIMIZATION}

The MSE for user $k$ is defined as

$$
\begin{aligned}
\varepsilon_{k} & =\mathrm{E}\left[\left|\hat{s}_{k}-s_{k}\right|^{2}\right] \\
& =\left|\boldsymbol{g}_{k}^{\mathrm{H}} \boldsymbol{H}_{k k} \boldsymbol{v}_{k}-1\right|^{2}+\sum_{\substack{j=1 \\
j \neq k}}^{K}\left|\boldsymbol{g}_{k}^{\mathrm{H}} \boldsymbol{H}_{k j} \boldsymbol{v}_{j}\right|^{2}+\left\|\boldsymbol{g}_{k}\right\|_{2}^{2} \sigma^{2}
\end{aligned}
$$

Instead of maximizing the total utility in (3), we first consider minimizing the sum MSE:

$$
\min _{\substack{\boldsymbol{v}_{1}, \ldots, \boldsymbol{v}_{K} \\ \boldsymbol{g}_{1}, \ldots, \boldsymbol{g}_{K}}} \sum_{k=1}^{K} \varepsilon_{k} \quad \text { s.t.: }\left\|\boldsymbol{v}_{k}\right\|_{2}^{2} \leq 1 \quad \forall k \in\{1, \ldots, K\} .
$$

The optimal receive filter for user $k$ is given by

$$
\boldsymbol{g}_{\mathrm{MMSE}, k}=\left(\sum_{j=1}^{K} \boldsymbol{H}_{k j} \boldsymbol{v}_{j} \boldsymbol{v}_{j}^{\mathrm{H}} \boldsymbol{H}_{k j}^{\mathrm{H}}+\sigma^{2} \mathbf{I}\right)^{-1} \boldsymbol{H}_{k k} \boldsymbol{v}_{k} .
$$

Optimizing the beamformers, subject to the transmit power constraint, gives

$$
\boldsymbol{v}_{\mathrm{MMSE}, k}=\left(\sum_{j=1}^{K} \boldsymbol{H}_{j k}^{\mathrm{H}} \boldsymbol{g}_{j} \boldsymbol{g}_{j}^{\mathrm{H}} \boldsymbol{H}_{j k}+\lambda_{k} \mathbf{I}\right)^{-1} \boldsymbol{H}_{k k}^{\mathrm{H}} \boldsymbol{g}_{k}
$$

where the Lagrange multiplier $\lambda_{k} \geq 0$ is chosen so that $\left\|\boldsymbol{v}_{k}\right\|_{2}^{2} \leq 1$. Note that given a set of receive filters, a particular user's beamformer does not depend on the other beamformers. An explicit expression for $\lambda_{k}$ cannot be given; it can be shown, however, that $\left\|\boldsymbol{v}_{\mathrm{MMSE}, k}\right\|^{2}$ is convex and decreasing in $\lambda_{k}$, so that there exists a unique solution, which can be efficiently found (e.g., via Newton iterations).

Based on the preceding observations, we propose the following iterative procedure for solving (10) (cf. [7]):

1) Initialize the beams $\boldsymbol{v}_{1}, \ldots, \boldsymbol{v}_{K}$ arbitrarily.

2) Optimize the receive filters $\boldsymbol{g}_{1}, \ldots, \boldsymbol{g}_{K}$ assuming fixed beamformers according to (11).

3) Optimize beamformers $\boldsymbol{v}_{1}, \ldots, \boldsymbol{v}_{K}$ assuming fixed receivers according to (12).

4) Repeat from 2) until convergence.

By noting that at each step the sum MSE is decreased or stays the same we have the following lemma.

Lemma 1: The sum MSE for the preceding algorithm converges. Moreover any stationary point corresponds to a local optimum. 
Comparing the transmitter and receiver updates (11) and (12) with those of the Max-SINR algorithm, (7) and (8), we notice that they are nearly identical. The two differences are the regularization with $\lambda_{k}$ instead of $\sigma^{2}$ inside the inverse in the beamformer update and the absence of the normalization factors $\theta_{k}$ and $\beta_{k}$.

\section{A. Weighted Sum MSE Minimization}

The MSE cost function can be easily extended to allow different priorities to be assigned to the users by means of weights $\alpha_{k}$, yielding the optimization problem:

$$
\min _{\substack{\boldsymbol{v}_{1}, \ldots, \boldsymbol{v}_{K} \\ \boldsymbol{g}_{1}, \ldots, \boldsymbol{g}_{K}}} \sum_{k=1}^{K} \alpha_{k} \varepsilon_{k} \quad \text { s. t.: } \quad\left\|\boldsymbol{v}_{k}\right\|_{2}^{2} \leq 1 \quad \forall k \in\{1, \ldots, K\} .
$$

Compared to the unweighted optimization problem in (10), the solution for the optimal receive filters remains the same (cf. (11)). The necessary condition for the beamformers must be modified as

$$
\boldsymbol{v}_{\mathrm{WMMSE}, k}=\left(\sum_{j=1}^{K} \frac{\alpha_{j}}{\alpha_{k}} \boldsymbol{H}_{j k}^{\mathrm{H}} \boldsymbol{g}_{j} \boldsymbol{g}_{j}^{\mathrm{H}} \boldsymbol{H}_{j k}+\lambda_{k} \mathbf{I}\right)^{-1} \boldsymbol{H}_{k k}^{\mathrm{H}} \boldsymbol{g}_{k}
$$

with $\lambda_{k} \geq 0$ so that $\left\|\boldsymbol{v}_{k}\right\|_{2}^{2} \leq 1$. Lemma 1 also applies to this extension.

\section{Weighted Sum MSE and General Sum Utilities}

We now use the weighted MSE optimization with adaptive weights to solve the original utility optimization problem (10). With MMSE receive filters given by (11), we note that the SINR $\gamma_{k}=\frac{1}{\varepsilon_{k}}-1$, so that we can express the utility functions in terms of the MSE, i.e.,

$$
\bar{u}_{k}\left(\varepsilon_{k}\right)=u_{k}\left(\frac{1}{\varepsilon_{k}}-1\right) .
$$

Expanding the sum utility in a Taylor expansion around the operating point $\varepsilon_{k, 0}$ and dropping all but the linear term gives

$$
\sum_{k=1}^{K} \bar{u}_{k}\left(\varepsilon_{k}\right)=\sum_{k=1}^{K}-\alpha_{k} \varepsilon_{k}+C+O\left(\epsilon_{k}^{2}\right)
$$

where $C$ does not depend on any $\varepsilon_{k}$ and

$$
\alpha_{k}=-\left.\frac{\partial \bar{u}_{k}\left(\varepsilon_{k}\right)}{\partial \varepsilon_{k}}\right|_{\varepsilon_{k}=\varepsilon_{k, 0}} .
$$

For example, the rate utility $u_{k}\left(\gamma_{k}\right)=\log \left(1+\gamma_{k}\right)$ becomes $\bar{u}_{k}\left(\varepsilon_{k}\right)=-\log \left(\varepsilon_{k}\right)$, so that the sum rate behaves locally as weighted sum MSE with weights $\alpha_{k}=1 / \varepsilon_{k, 0}$.

The resulting Adaptively Weighted-MSE (AW-MSE) algorithm follows:

1) Initialize the beamformers $\boldsymbol{v}_{1}, \ldots, \boldsymbol{v}_{K}$ arbitrarily, and compute the optimal receive filters $\boldsymbol{g}_{1}, \ldots, \boldsymbol{g}_{K}$ from (11) and the weights $\alpha_{1}, \ldots, \alpha_{K}$ from (17).

2) Iterately update the beamformers from (14) and the receivers from (11) until convergence. After each beamformer update, rescale the beamformer to have a unit norm.
3) Update the weights $\alpha_{1}, \ldots, \alpha_{K}$ according to the new operating point $\varepsilon_{1,0}, \ldots, \varepsilon_{K, 0}$ using (17).

4) Repeat from 2) until the weights $\alpha_{1}, \ldots, \alpha_{K}$ have converged.

The re-normalization in step 2 implies that $\lambda_{k}$ in (14) is chosen so that $\left\|\boldsymbol{v}_{k}\right\|_{2}^{2}=1$ (instead of an inequality constraint). Without this modification at high SNRs the algorithm tends to shut-off some users in a fully-loaded system, and does not achieve aligned solutions. ${ }^{3}$

When the algorithm has converged, clearly (11) and (14) (with the preceding modification) are fulfilled for all $k$. Furthermore, since the weights $\alpha_{k}$ 's correspond to the current value of $\varepsilon_{k}$, the necessary optimality conditions for the original sum utility maximization problem (3) are fulfilled, where the inequality power constraints are replaced with equality constraints. Hence if the algorithm converges, it finds a locally optimal solution. A similar algorithm has been proposed for MIMO broadcast channels in [8].

\section{A. Convergence}

Conditions under which the AW-MSE algorithm converges are stated in the following proposition.

Proposition 1: If $u_{k}$ satisfies $\left(\gamma_{k}+1\right) u_{k}^{\prime \prime}+2 u_{k}^{\prime} \geq 0$, or equivalently, $\bar{u}_{k}^{\prime \prime} \geq 0$ for all feasible $\gamma_{k}$ or $\varepsilon_{k}$ and for each $k$, then the sum utility given by the AW-MSE algorithm converges.

Proof: The proof consists of showing that updating the beamformers and receive filters, given new (updated) weights, will increase the sum utility relative to that before the last weight update. This is true because the condition $\bar{u}_{k}^{\prime \prime} \geq 0$ implies that the utility function is a convex function of the MMSE, so that the linearization of the objective in (16) lower bounds the sum utility objective and is tight at the current operating point, i.e.,

$$
\sum_{k=1}^{K} \bar{u}_{k}\left(\varepsilon_{k}\right) \geq \sum_{k=1}^{K} \bar{u}_{k}\left(\varepsilon_{k, 0}\right)-\sum_{k=1}^{K} \alpha_{k}\left(\varepsilon_{k}-\varepsilon_{k, 0}\right)
$$

where $\varepsilon_{k, 0}$ is user $k$ 's MMSE at the current operating point.

Given a set of new weights, $\alpha_{k}$ 's, the users then update their beamformers and receive filters according to step 2. After those updates we must have

$$
\sum_{k=1}^{K} \alpha_{k} \varepsilon_{k, *}<\sum_{k=1}^{K} \alpha_{k} \varepsilon_{k, 0}
$$

where $\epsilon_{k, *}$ is the updated MSE for user $k$. Combining (19) and (18) implies that

$$
\sum_{k=1}^{K} \bar{u}_{k}\left(\varepsilon_{k, *}\right) \geq \sum_{k=1}^{K} \bar{u}_{k}\left(\varepsilon_{k, 0}\right)
$$

\footnotetext{
${ }^{3}$ Of course, this modification may not be reasonable when the number of users is greater than the number that can be aligned; our current work is considering algorithms that achieve alignment without this restriction.
} 


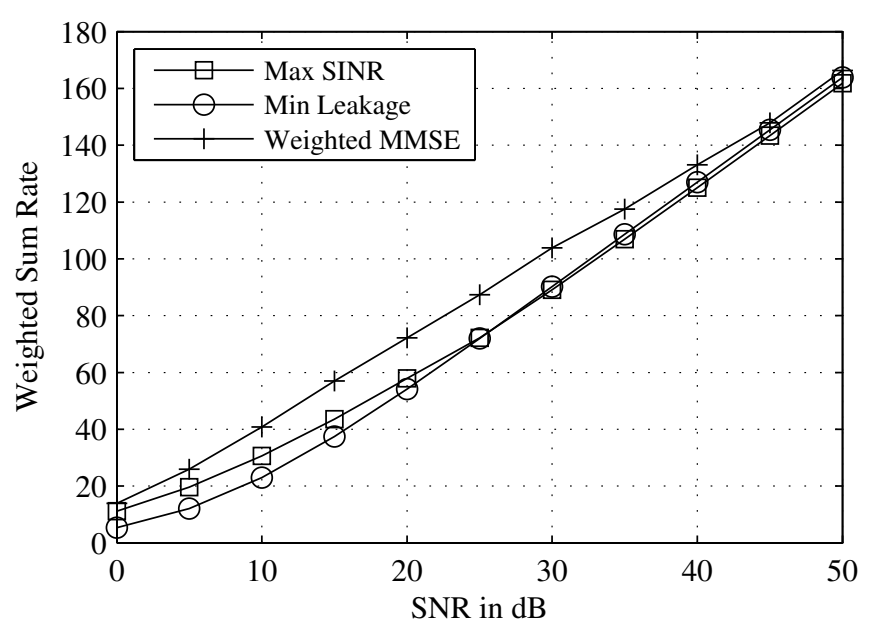

Fig. 2. Performance of distributed algorithms with three users and $2 \times 2$ channels.

or equivalently,

$$
\sum_{k=1}^{K} u_{k}\left(\gamma_{k, *}\right) \geq \sum_{k=1}^{K} u_{k}\left(\gamma_{k, 0}\right),
$$

which means the sum utility cannot decrease after the beamformers and receive filters are updated. Since the sum utility is bounded, it must therefore converge.

The proof applies when the weights, beamformers, and receive filters are updated asynchronously, as long as the receive filters are optimized for the current set of beams before each weight update. That is, (19) still holds, which guarantees that the sum utility cannot decrease over consecutive weight updates. The condition in Proposition 1 applies to the rate utility $\log (1+\gamma)$, but excludes the $\alpha$-fair utility $\gamma^{\alpha} / \alpha$. Although the preceding proof implies convergence of the sum utility objective, and not the beamformers, convergence of the beams is always observed in simulations.

\section{NumericAl Results}

In this section, we present numerical examples for a network of three users with $2 \times 2$ channels. The utility functions are weighted rates, i.e., $u_{k}\left(\gamma_{k}\right)=w_{k} \log _{2}\left(1+\gamma_{k}\right)$, where $w_{1}=0.1, w_{2}=1$, and $w_{3}=10$, reflecting an increase in priorities for users 1,2 , and 3 . The direct- and crosschannel gains are all i.i.d. with unit variance. Fig. 2 shows the weighted sum utility averaged over 100 channel realizations. Results are shown for the AW-MSE algorithm, Max-SINR, and Min-Leakage algorithm. At moderate SNRs the AW-MSE shows a significant gain in the objective, relative to the other algorithms, whereas at high SNRs all three algorithms exhibit similar performance, since alignment becomes optimal.

Fig. 3 shows achievable points in the rate region obtained by varying the MSE weights in the AW-MSE algorithm. This example is for a particular channel realization with an SNR of $10 \mathrm{~dB}$. Two additional points are shown corresponding to the Max-SINR and min-leakage algorithms. Those are connected

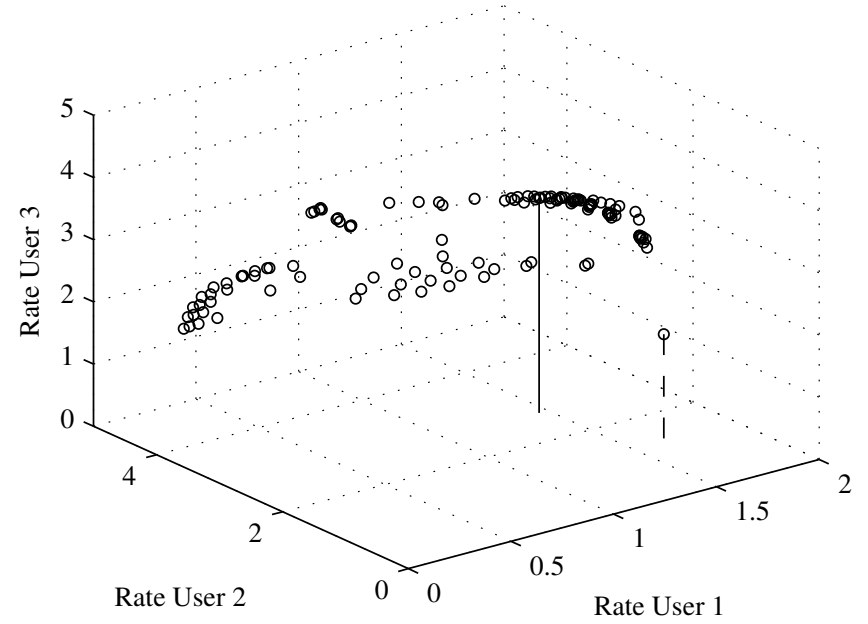

Fig. 3. Different Points in the Achievable Region

to the bottom plane by solid and dashed lines, and essentially correspond to the two aligned solutions. Further examples show that as the SNR increases, the points tend to cluster around the dominant aligned solution.

\section{CONCLUSIONS}

We have presented a distributed algorithm for adjusting beamformers in MIMO networks to maximize the sum utility over all users. Each beamformer is updated to minimize a weighted sum MSE objective, where the weights are updated according to the user utilities. Numerical examples show that this algorithm achieves alignment at high SNRs, and for the sum rate utility performs essentially the same as the MaxSINR algorithm in [5]. Moreover, it can achieve different points on the rate region by changing the MSE weights, and has provable convergence. A topic for further study is to relax the assumption of rank-one precoders, and allow for multiplexing gains on each link.

\section{REFERENCES}

[1] V. R. Cadambe and S. A. Jafar, "Interference Alignment and the Degrees of Freedom for the K User Interference Channel," IEEE Trans. Inform. Theory, vol. 54, no. 8, pp. 3425-3441, Aug. 2008.

[2] J. Huang, R. A. Berry, and M. L. Honig, "Distributed Interference Compensation for Wireless Networks," IEEE Journal on Selected Areas in Communications, vol. 24, no. 5, pp. 1074-1084, May 2006.

[3] D. A. Schmidt, C. Shi, R. A. Berry, M. L. Honig, and W. Utschick, "Pricing Algorithms for Power Control and Beamformer Design in Interference Networks," IEEE Signal Processing Magazine, September, 2009.

[4] C. Shi, D. A. Schmidt, R. A. Berry, M. L. Honig, and W. Utschick, "Distributed Interference Pricing for the MIMO Interference Channel," in Proc. IEEE International Conference on Communications, June 2009.

[5] K. S. Gomadam, V. R. Cadambe, and S. A. Jafar, "Approaching the Capacity of Wireless Networks through Distributed Interference Alignment," in Proc. of IEEE GLOBECOM, Nov./Dec. 2008.

[6] C. M. Yetis, T. Gou, S. A. Jafar, A. H. Kayran, "Feasibility Conditions for Interference Alignment", in Proc. of IEEE GLOBECOM, Nov. 2009.

[7] S. Serbetli and A. Yener, "Iterative Transceiver Optimization for Multiuser MIMO Systems," in Proc. Allerton Conf., Oct. 2002.

[8] R. Agarwal, S. S. Christensen, E. de Carvalho, and J. Cioffi, "Weighted Sum-Rate Maximization using Weighted MMSE for MIMO-BC Beamforming Design," IEEE Trans. on Wireless Comm., vol. 7, no. 12, pp. 1-8, Dec. 2008. 